\title{
The Journey of Clinical Registries Through Various Phases of the Digital Age: A Technical Perspective
}

\author{
Mansoor Ali BAIG ${ }^{\mathrm{a}, \mathrm{b}, 1}$, Mohammad Shouki BAZARBASHI ${ }^{\mathrm{a}}$, Haifa AlDakhil ${ }^{\mathrm{a}}$ and \\ Saniyah M. BAIG ${ }^{\mathrm{c}}$ \\ ${ }^{a}$ King Faisal Specialist Hospital \& Research Center, Riyadh, Saudi Arabia \\ ${ }^{b}$ Department of Biostatistics Epidemiology \& Scientific Computing, KFSHRC, KSA \\ ${ }^{c}$ Ilia State University, Tbilisi, Georgia
}

\begin{abstract}
The concept of registry-based medical research goes back to the mid of 18 th century where data was collected in actual physical registers and analyzed using manual counts in a very primitive way until computing technologies took over to digitize information, to change the process all the way from data collection to data analysis. This digital age of technology can be hypothetically classified in 3 eras; the Digitization Era, the Integration Era, and finally the Futuristic, Smart Intelligence Era. This study would highlight the changes in the fundamental aspects of a medical registry under each of these digital eras.
\end{abstract}

Keywords. Clinical registries, Disease registries, healthcare data, EDC

\section{Introduction}

The National Leprosy Registry, in Norway represented the world's first national patient register for any disease, established in 1856 [1], and eventually it became a practice to establish various disease registry programs to carry out evidence-based clinical research. These data repositories played a significant role in the control of the disease by evaluating the trends in disease incidence and other characteristics and thus helping the providers to plan accordingly. Medical registries have been identified as a source of information and evidence for patient monitoring, promoting evidence-based clinical decision-making [2]. Coupled with advancements in tools and technology, there has been a significant improvement and development in the way the registries are managed. It is worth studying and benchmarking the achievements of each phase of these advancements in relation to the other.

\section{Methods}

This study involves observation of various aspects of a medical registry under different progressive time frames (digital eras). These aspects not only include the collection,

${ }^{1}$ Corresponding Author, Mansoor Ali BAIG, BESC, King Faisal Specialist Hospital \& Research Center, Riyadh, Saudi Arabia; E-mail: mansoorbaig@hotmail.com, Mbaig97@kfshrc.edu.sa. 
entry, storage, extraction, processing, audit, reporting, analysis and integration of data, but also include predictive and prescriptive modeling of data. These aspects may be common to all eras or may be specific to a particular era.

\section{Results}

The digital revolution which started during the end of the $20^{\text {th }}$ century, fueled the exponential growth of data. This revolution also created a great demand for advancements in tools and techniques to simplify data collection, storage and extraction. Ease in accessibility and availability of research quality data along with simplified, seamless and unified access (locally as well as on global platforms) was desired as the outcome of these advancements. This exponential growth in the data digitization efforts can be categorized into 3 major digitization eras, namely the Early Digitization Era, the Centralization or Integration Era and the Futuristic Smart Intelligence Era. This study would highlight the changes in the fundamental aspects of a medical registry under each of these digital eras.

\subsection{Early Digitization Era}

This was the most critical and cumbersome phase and the first step towards transformation to a modern age, where one had to give up the traditional way of doing things as the modern system would demand a 360-degree change as to how things would be done.

In the early phases, the registries had to run a parallel process along with the traditional methods to carry out the data collection, storage, extraction and analysis. Primitive systems were developed for the electronic data capture, which required manpower with computing skills, which were scarce during the early phase. The paper based case report forms were still used as a primary data capture instrument, and later as an instrument to audit or validate the electronic data. Certain issues like system portability, distributed data sourcing and data availability was more of a challenge. In this phase, the data was collected on paper based instruments (case report forms), verified by the research registrar and manually entered in the electronic data capture system. A random audit of the electronic data was carried out, which was also based on the primary data capture instrument. While on the other hand, it was very difficult or in some cases almost impossible to verify or audit the primary data, which was collected on paper. It took a lot of time and effort to streamline and adapt towards an electronic data capture system to manage and maintain the registry data. Some of the ancient registry programs had an additional burden of transforming the retrospective data. A few followed the traditional data entry approach and a few tried to transform or rather extract their paper based data using the OCR (Optical character recognition) technology [3].

The electronic data capture systems were an interface to a data repository, which was either maintained as a file based system or a database management system (DBMS) or a relational database management system (RDBMS), whereas, some registries mostly with limited or linear dataset (flat format) preferred to capture their data in a spreadsheet to avoid the complicated data extraction step and ease the use of carrying out the data analysis and reporting. Lastly, the data collected had to be reported as well as analyzed to provide the necessary evidence for the research hypothesis. In the early age, some primitive 3rd party data analysis tools were available, but mostly the EDC would provide 
predesigned as well as custom reporting tools which had the capability of carrying out the basic analytics on the fly.

During this era, the data was digitized and stored in a silo with a closed system which did not have the ability to communicate or exchange data with other systems or entities.

\subsection{Data Integration Era:}

With a rapid growth in the internet penetration, it was now possible to move, transfer, interlink, interchange, interact with and within the data repositories, following an integrated approach. During this era, there were various governmental mandates on healthcare providers to quickly adapt to a Healthcare Information System (HIS) in order to maintain an electronic health record for the patients. During this period, data was still being captured redundantly in the Electronic registry as well as the HIS. During the primary phase of HIS implementations, the quality of data was not trusted upon and besides this, there were various technical and ethical issues with access to the HIS data, but during the mid-phase most organizations facilitated access to the HIS, which was used by the registries as a secondary data source, mostly to carry out data audits. But in the later phases, the registries collaborated with the medical records, medical informatics and the IT teams to come up with various data integration methods and strategies to avoid or minimize manual data capture. New methods/tools were introduced to capture the data directly from the data warehouse or. importing the most common minimum dataset from existing registries [2], or setting up an online-offline model to download the required data to be manually imported in the registry database.

Dynamic reporting features were made available, as a part of the registry systems. Also a number of sophisticated data analysis and bio-statistical tools like IBM SPSS, SAS, STATA etc. were available for carrying out any detailed and advanced data analysis.

\subsection{The Smart Intelligence Era:}

We have currently approached the beginning of this era, where various integrated systems communicate seamlessly through minimal human intervention, in order to manage and maintain the required data through a single interface deployed on an integrated environment. Number of cohorts can be run on the fly to identify or validate a research hypothesis, based on which the data can be sourced. These systems would also take a data based approach to generate various research hypothesis, each of which could be taken up as a distinct research project.

The concept of a silo based registry need not be followed and instead HIS itself can be a primary source once the necessary tools, software or platforms are implemented. These tools would act as an honest broker [4]; taking care of the good clinical practice. Further, based on the IRB approval, the required data can be sourced.

With a vision of establishing and intelligent enterprise, some healthcare entities have embraced the concept of a Data Science Hub [9] to assist researchers with their data problems, covering the descriptive, predictive and prescriptive approaches.

Systems like I2B2 [5] can be very instrumental in running cohorts on the HIS data to speed up the research process. I2B2 SHRINE [6] is another platform which helps healthcare organizations around the globe to share de-identified data or cohorts, enabling the healthcare providers to carry out disease specific benchmarking studies or validate their research hypothesis. Another futuristic approach by CLINERION [8] is to globalize 
or rather centralize the de-identified clinical data (in form of counts), to be made available primarily to the pharma companies to globally identify patient samples through discovery cohorts in order to carry out phase $1 / 2 / 3$ clinical trials. This same approach can be extended towards registries. Such initiatives accelerate development of innovative medical treatments by improving efficiency in clinical research.

The later phases of this era would be futuristic and based on Machine Learning, AI and collaborative research helping the global healthcare community, carry out benchmarking studies and personalized care. A registry system in the near future would just be a virtual interface to a data view generated through a well-organized data mart arranged through integrated systems, further integrated with advanced AI enabled analytical engines [7] where not only descriptive, but even predictive analysis could be done on the fly. These advancements in technology would act as a vital catalyst in speeding up research based activities in any healthcare setup, drastically reducing the research turnaround time, ensuring the highest quality coupled with an innovative approach.

\section{Conclusion}

The study provides a progressive view of the various aspects of registries in the light of the different phases of the digital age and also anticipates the possible advancements and implications of the concept of registries in the future. This study also highlights some unique platforms which works towards globalization of healthcare data along with strict adherence to the ethics and good clinical practice. These advanced tools and platforms would eventually virtualize the concept of managing and maintaining registry data or a registry based system. It would also provide a tool to the providers to define and design a registry in a matter of days if not hours.

\section{References}

[1] Irgens LM. The origin of registry-based medical research and care. Acta Neurol Scand Suppl. 2012;(195):4-6. doi: 10.1111/ane.12021. PMID: 23278649.

[2] Baig MA, Alzahrani SJ, Almuhaizea MA, Al-Shagathrh F. Fundamentals of Implementing a National Clinical Registries Program. Stud Health Technol Inform. 2019 Jul 4;262:384-387. doi: 10.3233/SHTI190099. PMID: 31349248.

[3] Paulsen A, Harboe K, Dalen I. Data entry quality of double data entry vs automated form processing technologies. Health Sci Rep. 2020 Nov 29;3(4):e210. doi: 10.1002/hsr2.210. PMID: 33283058

[4] Choi, H. J. et al. (2015). Establishing the role of honest broker: bridging the gap between protecting personal health data and clinical research efficiency. PeerJ, 3, e1506. https://doi.org/10.7717/peerj.1506

[5] Klann, J. G. et. al. (2016). Data interchange using i2b2. Journal of the American Medical Informatics Association: JAMIA, 23(5), 909-915. https://doi.org/10.1093/jamia/ocv188

[6] McMurry et. al. (2013). SHRINE: enabling nationally scalable multi-site disease studies. PloS one, 8(3), e55811. https://doi.org/10.1371/journal.pone.0055811

[7] PAT Research, Top 18 Artificial Intelligence Platforms, 2020.

[8] https://www.predictiveanalyticstoday.com/artificial-intelligence-platforms

[9] Clinerion,[n.d] Ideas and insights from our clinical research and real-world data experts

[10] https://www.clinerion.com/index/Experts-Corner

[11] Baig MA, Alzahrani SJ. Developing a Framework for a Healthcare Data Science Hub; Challenges and Lessons Learned. Stud Health Technol Inform. 2019. doi: 10.3233/SHTI190008. PMID: 31349257. 